\title{
Negative argininosuccinate synthetase expression in melanoma tumours may predict clinical benefit from arginine-depleting therapy with pegylated arginine deiminase
}

\section{LG Feun",', A Marini', G Walker', G Elgart', F Moffat', SE Rodgers', CJ Wu', M You', M Wangpaichitr', MT Kuo ${ }^{2}$, W Sisson ${ }^{3}$, AA Jungbluth ${ }^{4}$, J Bomalaski ${ }^{3}$ and N Savaraj'}

'Sylvester Comprehensive Cancer Center, University of Miami, 1475 NW 12th Avenue, Miami, FL 33136, USA; ${ }^{2}$ MD Anderson Cancer Center, 1515 Holcombe Boulevard, Houston, TX 77030, USA; ${ }^{3}$ Polaris Group, 6379 Nancy Ridge Drive, San Diego, CA 92121 , USA; ${ }^{4}$ Ludwig Institute for Cancer Research at Memorial Sloan Kettering Cancer Center, 1275 York Avenue, New York, NY I002I, USA

BACKGROUND: Arginine-depleting therapy with pegylated arginine deiminase (ADI-PEG20) was reported to have activity in advanced melanoma in early phase I-II trial, and clinical trials are currently underway in other cancers. However, the optimal patient population who benefit from this treatment is unknown.

METHODS: Advanced melanoma patients with accessible tumours had biopsy performed before the start of treatment with ADIPEG20 and at the time of progression or relapse when amenable to determine whether argininosuccinate synthetase (ASS) expression in tumour was predictive of response to ADI-PEG20.

RESULTS: Twenty-seven of thirty-eight patients treated had melanoma tumours assessable for ASS staining before treatment. Clinical benefit rate (CBR) and longer time to progression were associated with negative expression of tumour ASS. Only I of 10 patients with ASS-positive tumours (ASS +) had stable disease, whereas 4 of 17 (24\%) had partial response and 5 had stable disease, when ASS expression was negative (ASS -), giving CBR rates of 52.9 vs $10 \%, P=0.04$ I. Two responding patients with negative ASS expression before therapy had rebiopsy after tumour progression and the ASS expression became positive. The survival of ASS - patients receiving at least four doses at $320 \mathrm{IUm}^{-2}$ was significantly better than the ASS + group at 26.5 vs 8.5 months, $P=0.024$.

CONCLUSION: ADI-PEG20 is safe and the drug is only efficacious in melanoma patients whose tumour has negative ASS expression. Argininosuccinate synthetase tumour positivity is associated with drug resistance and tumour progression.

British Journal of Cancer (2012) 106, |48I-1485. doi:10.1038/bjc.2012.106 www.bjcancer.com

Published online 3 April 2012

(C) 2012 Cancer Research UK

Keywords: melanoma; arginine; arginine deiminase

Despite new agents, such as BRAF inhibitors and ipilimumab, the prognosis for most advanced melanoma patients remains poor and new therapies are still needed. Amino-acid depletion has been used to treat certain malignancies (Pinheiro and Boos, 2004). One such nonessential amino acid is arginine (Tapiero et al, 2002; Wheatley, 2004). Arginine is synthesised from citrulline via the enzyme argininosuccinate synthetase (ASS; Husson et al, 2003; Shen et al, 2003). Several tumours such as melanoma do not express ASS and are unable to synthesise arginine (Takaku et al, 1992; Ensor et al, 2002; Park et al, 2003; Dillon et al, 2004; Szlosarek et al, 2006; Kim et al, 2007). Pegylated arginine deiminase (ADI-PEG20), which degrades arginine to citrulline, has entered into clinical trials (Holtsberg et al, 2002; Curley et al, 2003; Izzo et al, 2004; Ascierto et al, 2005; Feun et al, 2008a; Ott et al, 2009; Dinh et al, 2011). ADI-PEG20 has shown activity in melanoma in a phase I/II trial. The optimal biologic dose (OBD) sufficient to eliminate all

*Correspondence: Dr LG Feun; E-mail: Ifeun@med.miami.edu

Presented in part at the 46th Annual Meeting of the American Society of Clinical Oncology, 4-8 June 2010.

Received 6 January 2012; revised 24 February 2012; accepted 6 March 2012; published online 3 April 2012 detectable arginine from the circulation for at least 7 days was $160 \mathrm{IU} \mathrm{m}^{-2} \mathrm{IM}$ given weekly. To investigate the role of ASS expression with tumour response, we conducted a clinical/ pharmacodynamic study of ADI-PEG20.

\section{MATERIALS AND METHODS}

Inclusion criteria included: (1) histologically confirmed metastatic melanoma, (2) unresectable and measurable/evaluable disease, (3) progressive disease after chemotherapy, radiotherapy, surgery or immunotherapy, no longer responding to standard therapy or have refused standard therapy, (4) been off previous treatment for at least 4 weeks, (5) fully recovered from prior surgery, (6) age $\geqslant 18$ years old, (7) KPS $\geqslant 70,(8)$ expected survival of $\geqslant 12$ weeks, (9) absolute neutrophil count $>1500$ per $\mu$, platelets $>100000$ per $\mu \mathrm{l}$, serum bilirubin $\leqslant 3 \mathrm{mg} \mathrm{dl}^{-1}$, albumin $>3 \mathrm{~g} \mathrm{dl}^{-1}$, alkaline phosphatase $<5 \times \mathrm{ULN}$, glucose $>60 \mathrm{mg} \mathrm{dl}^{-1}$, amylase $<1.5 \times$ ULN, (10) signed an IRB approved informed consent, and (11) not enroled in other IND studies. Patients with metastatic ocular melanoma were allowed but analysed separately. Exclusion criteria included: (1) significant cardiac disease (New York Heart Association Class 3 or 4), (2) serious infection requiring 
antibiotics, (3) pregnancy or lactation, (4) clinical ascites or symptomatic pleural effusion, (5) expected non-compliance, and (6) known allergy to other Escherichia coli drug products (such as GM-CSF). Patients with stable brain metastases were not excluded. Patients continued to receive treatment until: (1) the patient developed progressive disease or (2) the patient had grade 3 or greater toxicity, except for local discomfort from injection or (3) the patient had grade 2 or greater allergic reaction or (4) the patient had a complete response. There was no dose de-escalation for toxicity. If a complete response was observed, the patient continued treatment for additional 4 weeks. If the patient had stable disease or less than a complete response, the patient was allowed to continue therapy as long as no major toxicity was observed. There was no maximum number of cycles required. Analysis of clinical outcomes was done by Sylvester Cancer Centre following closure of the study by the sponsor after 39 patients had been enroled and treated. Clinical outcome data include best overall response, disease progression and survival. ADI-PEG20 was administered at weekly intramuscular doses. The initial starting dose for the protocol was $160 \mathrm{IU} \mathrm{m}^{-2}$. When it was noted that $160 \mathrm{IU} \mathrm{m}^{-2}$ did not decrease peripheral blood arginine to nondetectable levels, the dose was increased to $320 \mathrm{IU} \mathrm{m}^{-2}$. Twenty-one patients received a starting dose of $160 \mathrm{IU} \mathrm{m}^{-2}$, based on the OBD. One cycle of therapy was considered 4 weekly doses. Patients had radiologic evaluation, CT scan after 2 months and every 2 months while on study. If they tolerated the drug well (without grade 3 or greater toxicity or grade 2 allergic reaction) and clinically have stable disease or better, they were given another two cycles. Dose escalation (up to $320 \mathrm{IU} \mathrm{m}^{-2}$ ) was allowed in patients who had stable disease. Patients who had progressive disease after two cycles of therapy were taken off study. Fifteen patients with cutaneous melanoma and two patients with ocular melanoma received a starting dose of $320 \mathrm{IU} \mathrm{m}^{-2}$. Biopsy of accessible tumour was performed whenever possible for determination of ASS expression. At relapse, accessible tumour was obtained if patients agreed. The RECIST criteria were used to determine response. Duration of response was measured from start of therapy to tumour progression. Response rates were estimated with corresponding $95 \%$ confidence intervals (CIs) by the binomial method. Progression-free survival (PFS) and overall survival (OS) were estimated by the Kaplan-Meier method with corresponding 95\% CIs for survival rates and median survival times based on Greenwood's variance and the log-log transform method. Progression-free survival was defined as the elapsed time from treatment start to earliest documented evidence of disease progression or death from any cause. Patients who remained alive without progression were treated as censored observations using the date of last disease assessment. Overall survival was defined as the elapsed time from treatment start to death from any cause with follow-up for surviving patients censored at the date of last contact. In the subset of patients for whom baseline ASS was measured, Fisher's exact test was used to compare response rate in ASS-positive $(\mathrm{ASS}+)$ vs ASS-negative (ASS - ) patients, and the log-rank test was used to compare PFS and OS.

ASS expression was performed by immunohistochemical staining (Dillon et al, 2004). The criteria for determining ASS positivity and negativity was as follows: normal endothelial cells in the section were used as positive control, any positive staining regardless of the intensity of staining was considered as positive. The reason is that our in-vitro data strongly suggest that for primary culture or cell lines with positive staining regardless of the intensity, ASS can be induced rather rapidly (Savaraj et al, 2007). Examples of ASS-positive melanoma and ASS-negative melanoma are shown in Supplementary Figures $1 \mathrm{~S}$ and $2 \mathrm{~S}$. When feasible, RT-PCR or qRT-PCR was performed in the primary culture (see Supplementary Method). At the beginning of the study, RT-PCR was performed (Savaraj et al, 2007) while we developed the assay for qRT-PCR (see Supplementary Method). Normal human fibroblasts (BJ-1) which express ASS and are resistant to ADIPEG20 were used as a positive control and the value was set at one. The arbitrary value of $<0.01$ was considered as negative (Dinh et al, 2011).

\section{RESULTS}

\section{Patient characteristics}

A total of 39 patients were enroled and treated, 37 at the SCCC and 2 at the VA Medical Center. The primary analysis set consisted of 38 patients, excluding 1 patient deemed ineligible who received study treatment $<4$ weeks before the completion of radiation therapy.

The characteristics of the 38 patients who constitute the primary analysis set are given in Table 1 . In all, 2 patients had ocular and 36 had cutaneous melanoma. Of the 36 cutaneous melanoma patients, 2 failed to complete one cycle (4 weeks) of therapy and return for follow-up. One experienced trauma from a fall unrelated to the study drug and could not return weekly and was taken off study. One patient with malignant pleural effusion required a chest tube

Table I Characteristics of the 38 patients

\begin{tabular}{|c|c|}
\hline Characteristic & No. of patients (\%) \\
\hline \multicolumn{2}{|l|}{ Sex } \\
\hline Male & $25(66)$ \\
\hline Female & $13(34)$ \\
\hline Age, years median (range) & $69(29-85)$ \\
\hline \multicolumn{2}{|l|}{ AJCC stage at entry } \\
\hline Stage III & $2(5)$ \\
\hline Stage IV Mla & $5(13)$ \\
\hline Stage IV MIb & $8(21)$ \\
\hline Stage IV MIc & $23(61)$ \\
\hline \multicolumn{2}{|l|}{ Baseline KPS } \\
\hline 100 & $24(63)$ \\
\hline 90 & $9(24)$ \\
\hline 80 & $4(11)$ \\
\hline 70 & I (3) \\
\hline \multicolumn{2}{|l|}{ Baseline LDH } \\
\hline$>$ ULN & $12(32)$ \\
\hline Normal & $26(68)$ \\
\hline \multicolumn{2}{|l|}{ Primary site } \\
\hline Cutaneous & $36(95)$ \\
\hline Ocular & $2(5)$ \\
\hline \multicolumn{2}{|l|}{ ASS staining } \\
\hline Negative & $17(45)$ \\
\hline Positive & $10(26)$ \\
\hline Not assessed & $11(29)$ \\
\hline \multicolumn{2}{|c|}{ Sites of disease (some patients had multiple sites) } \\
\hline Visceral & $7(18)$ \\
\hline Liver & $10(26)$ \\
\hline Lung & $22(58)$ \\
\hline Nonvisceral & $29(76)$ \\
\hline \multicolumn{2}{|c|}{ Prior therapy (patients may have had more than one type of prior therapy) } \\
\hline Radiation therapy & $19(50)$ \\
\hline Chemotherapy & $14(37)$ \\
\hline Systemic vaccine or biologic therapy & $15(39)$ \\
\hline \multicolumn{2}{|l|}{ Cytokine therapy } \\
\hline Interleukin-2 & $4(11)$ \\
\hline Interferon & $10(26)$ \\
\hline
\end{tabular}

Abbreviations: $\quad \mathrm{AJCC}=$ American Joint Committee on Cancer; $\mathrm{ASS}=$ argininosuccinate synthetase; KPS = Karnofsky performance status; $\mathrm{LDH}=$ lactate dehydrogenase; ULN = upper limit of normal. 
and declined further therapy after the first two weekly doses. Both patients were included in the response analysis. Toxicity data were collected on all patients treated. In terms of dosing for ADI-PEG20 received, the median was 8 doses (two cycles) and the range was $2-71$ doses.

\section{ASS expression}

There was an association between ASS expression of the melanoma tissue obtained before treatment and the clinical benefit from ADI-PEG20 therapy. Pretreatment tumour samples were available in 27 cutaneous melanoma patients. The remaining patients either declined to have pretreatment biopsies performed or these were not available.

Although heterogeneity does occur, however, once staining is positive in certain cells, these cells will not respond to arginine deprivation and will continue to proliferate. Subset analysis of 27 patients with known baseline ASS status is presented in Table 2.

All of the responders in this study were ASS negative. In all, 4 of 17 ASS - patients achieved PR and 5 had SD, giving a clinical benefit rate (CBR) rate of $52.9 \%$, compared with no PRs and one SD among 10 ASS + patients. Although the difference in PR rate did not attain statistical significance, $23.5 \%$ vs $0 \%, P=0.264$, the difference in CBR did, 52.9 vs $10.0 \%, P=0.042$. The duration of $P R$ was $6,6+, 12$ and 16 months. One patient had PR of a cutaneous lesion and after 6 months had surgery to remove the lesion. Pathology showed no viable tumour, only melanosis. Sites of response included soft tissue/nodal (three patients) and lung (one patient). Interestingly, two patients who were ASS negative by immunohistochemistry and RT-PCR at the start of therapy had a response to ADI-PEG20. When these two patients developed progression of disease, repeat biopsy of the melanoma showed ASS positive. The first patient had lung metastases and had a PR to ADI-PEG20. At the time of progression, a lung resection showed ASS-positive tumour. The second patient had multiple subcutaneous masses and had a PR. Sixteen months later at the time of tumour progression, a repeat biopsy showed ASS positive (see Supplementary Data). One patient was ASS negative by immunohistochemical staining but qPCR value of 0.09 for ASS and failed to respond. Repeat biopsy was ASS positive by immunohistochemical staining at the time of failure. Two patients with ocular melanoma were treated. One patient was ASS positive and did not respond. The other patient did not have ASS expression tested and had mixed response. Overall for the entire population treated, 4 out of $36(11 \%)$ patients with cutaneous melanoma showed response, and the disease control rate including responding and stable patients (clinical benefit) was 10 out of $36(28 \%)$.

Negative baseline ASS was also associated with longer PFS, $P=0.025$ (log-rank test); the median time to progression for ASS - patients was 3.6 months (95\% CI: 1.6-12.7) compared with 1.8 months (95\% CI: 0.7-3.4) for ASS + patients. Median OS was also longer for ASS - patients, 14.6 months (95\% CI: 4.4 to $<$ not attained $>$ ) as compared with 9.3 months (95\% CI: 2.5 to $<$ not attained $>$ ) for ASS + patients; however, the difference was not statistically significant, $P=0.374$ (log-rank test).

Table 2 Best overall response of cutaneous melanoma patients by baseline ASS status

\begin{tabular}{|c|c|c|c|c|c|}
\hline \multirow[b]{2}{*}{ Best overall response } & \multicolumn{2}{|c|}{ ASS - } & \multicolumn{2}{|c|}{ ASS +} & \\
\hline & $\mathbf{N}$ & $\%$ & $\mathbf{N}$ & $\%$ & \\
\hline Partial response & 4 & 23.5 & 0 & 0 & $P=0.264$ \\
\hline Stable, including 4 pts with minor response & 5 & 29.4 & I & 10.0 & \\
\hline Clinical benefit & 9 & 52.9 & I & 10.0 & $P=0.041$ \\
\hline Progression, including 2 pts with mixed response & 8 & 47.1 & 9 & 90.0 & \\
\hline
\end{tabular}

Abbreviation: $\mathrm{ASS}=$ argininosuccinate synthetase.

\section{ADI-PEG20 dosing}

Fifteen patients had an initial starting dose of $320 \mathrm{IU} \mathrm{m}^{-2}$ as did the two ocular melanoma patients. Twenty-one patients with cutaneous melanoma received a starting dose of $160 \mathrm{IU} \mathrm{m}^{-2}$ (four of whom had dose escalation up to $320 \mathrm{IU} \mathrm{m}^{-2}$ ). Of the 19 cutaneous melanoma patients treated with $320 \mathrm{IU} \mathrm{m}^{-2}$ (either as starting dose or as escalated dose at any time), 4 had response, whereas none of the 17 patients had response when the dosage was always $<320 \mathrm{IU} \mathrm{m}^{-2}, P=0.65$ (Fisher's exact test). The incidence of ASS expression was similar in both groups (data not shown). However, the survival of ASS - patients receiving at least four doses at $320 \mathrm{IU} \mathrm{m}^{-2}$ was significantly better than the ASS + group at 26.5 vs 8.5 months, $P=0.024$. Thus, the dosage appears to be important, independent of ASS expression. Of the 10 cutaneous melanoma patients with ASS - tumours who received $320 \mathrm{IU} \mathrm{m}^{-2}$, $4(40 \%)$ had PR and 3 had SD with the CBR of $70 \%$. In contrast, of seven patients who were ASS negative and had doses $<320 \mathrm{IU} \mathrm{m}^{-2}$, no partial responses were observed and only two had stable disease/minor response, $P=0.08$ (Fisher's exact test). Taken altogether, the data suggest that a higher dose of ADIPEG20 may be needed to keep arginine levels down in the long term.

\section{Toxicity}

Serious side effects from ADI-PEG20 were uncommon. The most common side effect thought to be drug related was mild/moderate discomfort at the intramuscular injection site, which occurred in virtually all the patients but did not require discontinuation of therapy. Other side effects included grade 3 allergic reaction, occurring during the fourth cycle (one patient), grade 4 neutropenia and thrombocytopenia (one patient), grade 3 neutropenia (three patients), grade 3 anaemia (one patient) and grade 3 fatigue (two patients). Four of the five patients who had myelosuppression had prior radiation therapy or radiation therapy with chemotherapy. The patient who had grade 4 neutropenia and thrombocytopenia had recent prior cranialspinal radiation therapy.

\section{DISCUSSION}

This is the first clinical study to demonstrate that response to arginine-depleting therapy did correlate with lack of ASS expression, with the CBR being statistically significant between ASS-negative and ASS-positive patients. All the responders and five of six patients with stable disease occurred in the ASS-negative patients. Only one patient had stable disease when ASS was positive in the melanoma tumour. One limitation of this study is the small number of patients who had ASS staining performed. However, this is the first and only study to our knowledge that has investigated and correlated ASS staining with tumour response and CBR. As the CBR was statistically significant, we believe that future clinical trials with ADI-PEG20 should consider targeting only melanoma patients with negative ASS expression and so a future trial in ASS-negative melanoma patients is planned. In this regard, we plan a follow-up study in which only advanced melanoma patients whose tumours are ASS negative will be treated with ADI-PEG20 at $320 \mathrm{IU} \mathrm{m}^{-2}$. This will help to give more definitive results on the CBR in ASS-negative patients, while our preliminary study looked at both ASS-negative and ASS-positive patients. Currently, there are clinical trials in progress with ADI-PEG20 in hepatocellular carcinoma, small-cell lung carcinoma and mesothelioma. No clinical correlation has been performed yet in these tumours between clinical response and ASS expression. However, given our experience in melanoma patients with ADI-PEG20 and the scientific rationale for depleting arginine with this agent, it seems logical to target only patients with ASS-negative deficiency. 
Another important observation from our trial is that the dose of ADI-PEG20 used to deplete arginine may be important for response. In a previous phase I/II trial, the dose of $160 \mathrm{IU} \mathrm{m}^{-2}$ per week was considered to be the OBD, defined as the dose that lowered the blood arginine level to nondetectable levels (Ascierto et al, 2005). In that trial, all of the responders and those with stable disease were treated at a dose of $160 \mathrm{IU} \mathrm{m}^{-2}$ or higher. The response rate was $25 \%$ in 24 patients. Patients were entered into one of six cohorts (doses ranged from 40 to $640 \mathrm{IU} \mathrm{m}^{-2}$ per week). That trial did not state which cohort had most of the responses, which may partially explain the discrepancy with our results. Although $160 \mathrm{IU} \mathrm{m}^{-2}$ per week was considered the OBD, blood arginine levels do not necessarily reflect tissue arginine levels. In our phase II trial, all the responding patients had received ADI-PEG20 at $320 \mathrm{IU} \mathrm{m}^{-2}$ per week and no patient responded when the dose was less. The incidence of ASS expression was similar at both doses of ADI-PEG20. Interestingly, of the 10 patients who received a dose of $320 \mathrm{IU} \mathrm{m}^{-2}$ and were ASS negative, $4(40 \%)$ had a partial response and 3 had stable disease for a CBR of $70 \%$, as well as prolonged survival compared with ASS + patients. Thus, the dose of ADI-PEG20 may also be important to obtaining response. This may explain the different results in another phase I/II trial in melanoma in which the maximum dose was limited to $160 \mathrm{IU} \mathrm{m}^{-2}$ per week (Ott et al, 2009). No responses were observed but stable disease and time to tumour progression appeared to correlate with ASS expression. Taken together, the data suggest that higher doses of ADI-PEG20 than the OBD may be needed in certain ASS-negative patients for response and patients who benefit have tumours which are negative for ASS expression. Importantly, serious toxicity from ADI-PEG20 was uncommon. ADI-PEG20 has been well tolerated in clinical trials with other cancer types (Izzo et al, 2004; Ascierto et al, 2005; Feun et al, 2008a; Ott et al, 2009).

We have found re-expression of ASS occurs at the time of relapse in two patients, which could explain the cause of treatment failure. This finding also was observed in our laboratory in which ASS expression in certain melanoma cell lines can be induced on arginine deprivation (Savaraj et al, 2007; Dinh et al, 2011) and is one of the mechanisms of ADI-PEG20 resistance. To circumvent this form of resistance, it is important to understand how ASS is regulated. In this respect, we have found that c-Myc and HIF- $1 \alpha$ can positively and negatively regulate ASS, respectively (Tsai et al, 2009). None of the melanoma patients in this trial were studied for c-Myc expression but this will be studied in future protocols with ADI-PEG20.

The critical questions are what is the optimal methodology to detect ASS and why do not all ASS-negative melanoma patients respond to ADI-PEG20? Thus far, immunohistochemical staining proved to be the most suitable method, as $\mathrm{qRT}-\mathrm{PCR}$ requires primary culture due to contamination from normal cells which could obscure the results. Extrapolating from cell lines, qPCR is more sensitive and it appears that when ASS mRNA $<0.09$, the immunostaining in cell line is negative (Dinh et al, 2011). Whether higher mRNA copies may be another factor governing the sensitivity to ADI-PEG20 as well as the development of resistance is not clear and requires large number of patients' samples.
As for the second question, we have found that ASS-negative melanoma cells (immunostaining negative) appear to fall into three groups (Feun et al, 2004; Savaraj et al, 2007; Tsai et al, 2009; Savaraj et al, 2010). The first group includes melanoma cells that cannot be induced to express ASS, undergo autophagy but switch to apoptosis with arginine deprivation (Savaraj et al, 2010). The second group includes cells that stop proliferating, undergoing autophagy but will not switch to apoptosis for over 7 days. This group required higher dose for cell death and may represent the 'stable disease' seen in patients after ADI-PEG20. The third group includes melanoma cells that can undergo autophagy but rapidly express ASS after arginine depletion. Interestingly, this group may have higher copy of ASS mRNA. Overall, resistance to ADI-PEG20 can be due to: (1) autophagy and (2) induction of ASS (Savaraj et al, 2010). From our combined laboratory and clinical data, several approaches can be used to improve the efficacy of ADIPEG20. One approach is to combine agents that inhibit autophagy. Currently, only chloroquine has been extensively studied but the doses used to inhibit autophagy may be too toxic. Newer agents of this type can be explored. Another approach is to increase the apoptotic response by combining with other agents (Feun et al, 2008b; You et al, 2010; You et al, 2011). We have shown that combination of cisplatin (via the intrinsic apoptotic pathway) or TRAIL (via extrinsic pathway) with ADI-PEG20 increases cell death in vitro (You et al, 2010; You et al, 2011). Combination with TRAIL showed a greater enhancement effect as arginine deprivation results in increasing DR4 and DR5, both are key receptors for TRAIL (You et al, 2010). Furthermore, activation of caspase 8 by TRAIL also cleaves Beclin1 and ATG5 resulting in attenuation of autophagy (You et al, 2011) and thus, redirecting cells to apoptosis. Overall, a better understanding of the mechanisms of resistance may improve the therapeutic outcome of arginine deprivation treatment in ASS-negative tumours (Yoon et al, 2007; Bowles et al, 2008; Kim et al, 2009).

In summary, ADI-PEG20 has activity in malignant melanoma and is well tolerated. ASS expression in tumour is important in determining response to therapy. The dose required for response may exceed the OBD previously recommended. Further clinical trials in melanoma with ADI-PEG20 should be considered to be conducted only in ASS-negative patients, perhaps in combination therapy with cisplatin or TRAIL to potentiate the response.

\section{ACKNOWLEDGEMENTS}

This work was supported by NIH grant no. R01CA109578

\section{Conflict of interest}

Wes Sisson and John Bomalaski are employees of Polaris. The remaining authors declare no conflict of interest.

Supplementary Information accompanies the paper on British Journal of Cancer website (http://www.nature.com/bjc)

\section{REFERENCES}

Ascierto PA, Scala S, Castello G, Daponte A, Simeone E, Ottaiano A, Beneduce G, De Rosa V, Izzo F, Melucci MT, Ensor CM, Prestayko AW, Holtsberg FW, Bomalaski JS, Clark MA, Savaraj N, Feun LG, Logan TF (2005) Pegylated arginine deiminase treatment of patients with metastatic melanoma: results from phase I and II studies. J Clin Oncol 23: 7660-7668

Bowles TL, Kim R, Galante J, Parsons CM, Virudachalam S, Kung HJ, Bold RJ (2008) Pancreatic cancer cell lines deficient in argininosuccinate synthetase are sensitive to arginine deprivation by arginine deiminase. Int J Cancer 123: 1950-1955
Curley SA, Bomalaski JS, Ensor CM, Holtsberg FW, Clark MA (2003) Regression of hepatocellular cancer in a patient treated with arginine deiminase. Hepatogastroenterology 50: 1214-1216

Dillon BJ, Prieto VG, Curley SA, Ensor CM, Holtsberg FW, Bomalaski JS, Clark MA (2004) Incidence and distribution of argininosuccinate synthetase deficiency in human cancers: a method for identifying cancers sensitive to arginine deprivation. Cancer 100: 826-833

Dinh V, You M, Savaraj N, Wu C, Kuo M, Wangpaichitr M, Feun L (2011) Determining argininosuccinate synthetase (ASS) expression in patients 
with melanoma treated with arginine depleting therapy. Proc Am Soc Clin Oncol 29: 661s (abstract no. 10627)

Ensor CM, Holtsberg FW, Bomalaski JS, Clark MA (2002) Pegylated arginine deiminase (ADI-SS PEG20000 mw) inhibits human melanomas and hepatocellular carcinomas in vitro and in vivo. Cancer Res 62: 5443-5450

Feun L, Wu C, Clark M, Bomalask J, Holtsberg F, Wangpaichitr M, Robles C, Savaraj N (2004) Mechanism of antitumor effect of arginine deiminase-polyethylene (ADI-PEG20) and the possible mechanism of resistance in melanoma. Proc Am Assoc Can Res 45: 1054

Feun L, You M, Wu C, Wangpaichitr M, Kuo M, Savaraj N (2008a) Clinical and pharmacologic study of ADI-PEG in hepatocellular carcinoma. Proc Am Soc Clin Oncol 26: 15584

Feun L, You M, Wu CJ, Kuo MT, Wangpaichitr M, Spector S, Savaraj N (2008b) Arginine deprivation as a targeted therapy for cancer. Curr Pharm Des 14: 1049-1057

Holtsberg FW, Ensor CM, Steiner MR, Bomalaski JS, Clark MA (2002) Poly(ethylene glycol) (PEG) conjugated arginine deiminase: effects of PEG formulations on its pharmacological properties. J Control Release 80: $259-271$

Husson A, Brasse-Lagnel C, Fairand A, Renouf S, Lavoinne A (2003) Argininosuccinate synthetase from the urea cycle to the citrulline-NO cycle. Eur J Biochem 270: 1887-1899

Izzo F, Marra P, Beneduce G, Castello G, Vallone P, De Rosa V, Cremona F, Ensor CM, Holtsberg FW, Bomalaski JS, Clark MA, Ng C, Curley SA (2004) Pegylated arginine deiminase treatment of patients with unresectable hepatocellular carcinoma: results from phase I/II studies. J Clin Oncol 22: 1815-1822

Kim JH, Yu YS, Kim DH, Min BH, Kim KW (2007) Anti-tumor activity of arginine deiminase via arginine deprivation in retinoblastoma. Oncol Rep 18: $1373-1377$

Kim RH, Coates JM, Bowles TL, McNerney GP, Sutcliffe J, Jung JU, Gandour-Edwards R, Chuang FY, Bold RJ, Kung HJ (2009) Arginine deiminase as a novel therapy for prostate cancer induces autophagy and caspase-independent apoptosis. Cancer Res 69: 700-708

Ott PA, Carvajal RD, Pandit-Taskar N, Jungbluth AA, Hoffman E, Venhaus R, Pan L, Old L, Pavlick AC, Wolchok JD (2009) Phase I/II study of arginine deiminase (ADI-PEG20) in patients with advanced malignant nelanoma(MM). ASCO 27: 468s

Park IS, Kang SW, Shin YJ, Chae KY, Park MO, Kim MY, Wheatley DN, Min $\mathrm{BH}$ (2003) Arginine deiminase: a potential inhibitor of angiogenesis and tumour growth. Br J Cancer 89: 907-914
Pinheiro JP, Boos J (2004) The best way to use asparaginase in childhood acute lymphatic leukaemia-still to be defined? Br J Haematol 125: 117-127

Savaraj N, Wu C, Kuo M, You M, Wangpaichitr M, Robles C, Sepector S, Feun L (2007) The relationship of arginine deprivation, argininosuccinate synthetase and cell death in melanoma. Drug Target Insights 2: 119-128

Savaraj N, You M, Wu C, Wangpaichitr M, Kuo MT, Feun LG (2010) Arginine deprivation, autophagy, apoptosis (AAA) for the treatment of melanoma. Curr Mol Med 10: 405-412

Shen LJ, Lin WC, Beloussow K, Shen WC (2003) Resistance to the antiproliferative activity of recombinant arginine deiminase in cell culture correlates with the endogenous enzyme, argininosuccinate synthetase. Cancer Lett 191: 165-170

Szlosarek PW, Klabatsa A, Pallaska A, Sheaff M, Smith P, Crook T, Grimshaw MJ, Steele JP, Rudd RM, Balkwill FR, Fennell DA (2006) In vivo loss of expression of argininosuccinate synthetase in malignant pleural mesothelioma is a biomarker for susceptibility to arginine depletion. Clin Cancer Res 12: 7126-7131

Takaku H, Takase M, Abe S, Hayashi H, Miyazaki K (1992) In vivo antitumor activity of arginine deiminase purified from Mycoplasma arginini. Int J Cancer 51: 244-249

Tapiero H, Mathe G, Couvreur P, Tew KD (2002) I. Arginine. Biomed Pharmacother 56: 439-445

Tsai W-B, Aiba I, Lee S-Y, Feun L, Savaraj N, Kuo MT (2009) Resistance to arginine deiminase treatment in melanoma cells is associated with induced argininosuccinate synthetase expression involving c-Myc/ HIF-1a/Sp4. Mol Cancer Therap 8: 3223-3233

Wheatley DN (2004) Controlling cancer by restricting arginine availability-arginine-catabolizing enzymes as anticancer agents. Anticancer Drugs 15: 825-833

Yoon CY, Shim YJ, Kim EH, Lee JH, Won NH, Kim JH, Park IS, Yoon DK, Min BH (2007) Renal cell carcinoma does not express argininosuccinate synthetase and is highly sensitive to arginine deprivation via arginine deiminase. Int J Cancer 120: 897-905

You M, Savaraj N, Wangpaichitr M, Wu C, Kuo M, Feun L (2011) TRAIL enhances cytotoxicity of arginine depletion therapy in argininosuccinate synthetase-negative melanoma cells through interuption of autophagy via activation of caspase. Proc Am Association Cancer Res 52: 979 (abstract no 4096)

You M, Savaraj N, Wangpaichitr M, Wu C, Kuo MT, Varona-Santos J, Nguyen DM, Feun L (2010) The combination of ADI-PEG20 and TRAIL effectively increases cell death in melanoma cell lines. Biochem Biophys Res Commun 394: 760-766

This work is published under the standard license to publish agreement. After 12 months the work will become freely available and the license terms will switch to a Creative Commons Attribution-NonCommercial-Share Alike 3.0 Unported License. 